Pacific Journal of Mathematic 


\title{
POWER SERIES RINGS OVER DISCRETE VALUATION RINGS
}

\author{
JIMMY T. ARNOLD
}

\begin{abstract}
If $V$ is a discrete valuation ring with Krull dimension $m$, it is shown that the power series ring $V\left[\left[x_{1}, \cdots, x_{n}\right]\right]$ has Krull dimension $m n+1$.
\end{abstract}

Throughout the paper all rings are assumed to be commutative with identity and the ring $R$ is not considered to be a prime ideal of $R$. In [1] the author defines a ring to have the SFT (strong finite type) property if for each ideal $A$ of $R$ there exists a finitely generated ideal $B$ and a positive integer $k$ such that $B \cong A$ and $a^{k} \in B$ for each $a \in A$. It is shown in [1, Theorem 1] that if $R$ fails to have the SFT-property then the power series ring $R \llbracket \mathrm{Y} \rrbracket$ has infinite Krull dimension. On the other hand, if $D$ is a Prüfer domain with $\operatorname{dim} D=m$ and if $D$ has the SFT-property then $\operatorname{dim} D[Y]=m+1$ [2, Theorem 3.8]. Recall that a valuation ring $V$ with finite Krull dimension is discrete if and only if $P \neq P^{2}$ for each nonzero prime ideal $P$ of $V$ [5, pp. 190-192]. A valuation ring $V$ has the SFT-property if and only if it is discrete [2, Proposition 3.1]. Thus, if $V$ is a valuation ring and $\operatorname{dim} V=m$ then either $V$ is discrete and $\operatorname{dim} V \llbracket Y \rrbracket=m+1$ (this specific result was proved by Fields in [4, Theorem 2.7]) or $V$ is nondiscrete and $\operatorname{dim} V \llbracket Y \rrbracket=\infty$. For $\operatorname{dim} R=m$ the author asks in [1, p. 303] if either $\operatorname{dim} R \llbracket Y \rrbracket=m+1$ or $\operatorname{dim} R \llbracket Y \rrbracket=\infty$. We show that the answer is no for $\operatorname{ring} V \llbracket x_{1}, \cdots, x_{n-1} \rrbracket$, where $V$ is a discrete valuation $\operatorname{ring}$ with $\operatorname{dim} V \geqq 2$. Specifically, we prove the following theorem.

THEOREM. If $V$ is a discrete valuation ring with Krull dimension $m$ then the power series ring $V \llbracket x_{1}, \cdots, x_{n} \rrbracket$ has Krull dimension $m n+1$.

Proof. The proof is by induction on $m$ and the case $m=1$ is well-known since, in this case, $V$ is Noetherian (cf. Lemma 2.6 of [4]). Thus assume that $m \geqq 2$, that the theorem holds if $\operatorname{dim} V=m-1$, let $\operatorname{dim} V=m$, and suppose that $(0)=P_{0} \subset P_{1} \subset P_{2} \subseteq \cdots \subseteq P_{m}$ is the set of prime ideals of $V$. Throughout the proof $X$ denotes the set $\left\{x_{1}, \cdots, x_{n}\right\}$ of analytic indeterminates over $V, V \llbracket X \rrbracket$ denotes the power series ring $V \llbracket x_{1}, \cdots, x_{n} \rrbracket, p \in P_{1} \backslash P_{1}^{2}, W=V_{P_{1}}, U=V / P_{1}, F=W / P_{1} W$ and, even though $P_{1}=P_{1} W$, we write $\mathscr{P}$ to denote the ideal $P_{1} W$. We note that $W$ is a rank one discrete valuation ring with maximal ideal $\mathscr{P}=p W, F$ is the quotient field of $U$, and for each integer $k \geqq 1$ 
we have $P_{1}^{k+1} \subseteq p^{k} P_{1}$. If $\xi \in(W \llbracket X \rrbracket)_{W \backslash(0)}$ then there exists a nonzero element $a$ in $W$ such that $a \xi \in W \llbracket X \rrbracket$. But then $p a \xi \in V \llbracket X \rrbracket$ and $p a \in V$ so $\xi \in(V \llbracket X \rrbracket)_{V \backslash(0)}$. This shows that $(W \llbracket X \rrbracket)_{W \backslash(0)} \subseteq(V \llbracket X \rrbracket)_{V \backslash(0)}$ and the reverse containment is obvious so equality holds. It follows that the correspondence $Q \rightarrow Q \cap V \llbracket X \rrbracket$ is a bijection from the set

$$
\{Q \in \operatorname{Spec}(W \llbracket X \rrbracket) \mid Q \cap W=(0)\}
$$

to the set $\left\{Q^{\prime} \in \operatorname{Spec}(V \llbracket X \rrbracket) \mid Q^{\prime} \cap V=(0)\right\}$ which preserves set containment. Thus, if $Q \in \operatorname{Spec}(W \llbracket X \rrbracket)$ and $Q \cap W=(0)$, then $\operatorname{rank} Q=$ $\operatorname{rank}(Q \cap V \llbracket X \rrbracket)$ and it follows that $\operatorname{rank} Q^{\prime} \leqq n$ for each $Q^{\prime} \in$ $\operatorname{Spec}(V \llbracket X \rrbracket)$ such that $Q^{\prime} \cap V=(0)$.

Let $(0) \subset Q_{1} \subset \cdots \subset Q_{t}=P_{m}+(X)$ be a maximal chain of prime ideals of $V \llbracket X \rrbracket$ and choose $k$ so that $Q_{k} \cap V=(0)$ while $Q_{k+1} \cap V \neq$ (0). Then, as we have already observed, $k=\operatorname{rank} Q_{k} \leqq n$. Since $p \in Q_{k+1} \quad$ we have $\left(P_{1} \llbracket X \rrbracket\right)^{2} \subseteq P_{1}^{2} \llbracket X \rrbracket \subseteq p P_{1} \llbracket X \rrbracket \subseteq Q_{k+1}$ and hence $Q_{k+1} \supseteqq P_{1} \llbracket X \rrbracket$.

We first consider the case in which $Q_{k+1} \neq P_{1} \llbracket X \rrbracket$. It follows from Theorem 3.14 of [3] that there exist elements $\lambda_{1}=x_{1}, \lambda_{2}, \cdots, \lambda_{n}$ in $x_{1} F \llbracket x_{1} \rrbracket$ such that the $U \llbracket x_{1} \rrbracket$-homomorphism $\phi: U \llbracket X \rrbracket \rightarrow U \llbracket \lambda_{1}, \cdots, \lambda_{n} \rrbracket$ determined by $\phi\left(x_{i}\right)=\lambda_{i}, 1 \leqq i \leqq n$, is an isomorphism. But $\phi$ extends to an $F \llbracket x_{1} \rrbracket$-epimorphism $\bar{\phi}: F \llbracket X \rrbracket \rightarrow F \llbracket x_{1} \rrbracket$ and if $\bar{Q}$ is the kernel of $\bar{\phi}$ then depth $\bar{Q}=1, \operatorname{rank} \bar{Q}=n-1$ [6, Corollary 1, p. 218], and $\bar{Q} \cap U \llbracket X \rrbracket=(0)$. Since $F \llbracket X \rrbracket=(W / \mathscr{P}) \llbracket X \rrbracket \cong W \llbracket X \rrbracket / \mathscr{P} \llbracket X \rrbracket$ and $U \llbracket X \rrbracket \cong$ $V \llbracket X \rrbracket / P_{1} \llbracket X \rrbracket, \bar{Q}$ determines a prime ideal $Q$ of $W \llbracket X \rrbracket$ such that depth $Q=1, \operatorname{rank}(Q / \mathscr{P} \llbracket X \rrbracket)=n-1$, and $Q \cap V \llbracket X \rrbracket=P_{1} \llbracket X \rrbracket$. Therefore, $\operatorname{rank} Q \geqq n$ and, since $\operatorname{dim} W \llbracket X \rrbracket=n+1$, it follows that rank $Q=n$. If we choose $f_{1}, \cdots, f_{n-1} \in X W \llbracket X \rrbracket$ such that the corresponding elements $\bar{f}_{1}, \cdots, \bar{f}_{n-1}$ in $F \llbracket X \rrbracket$ form a regular system of parameters for $(F \llbracket X \rrbracket)_{\bar{Q}}$, then $\left\{f_{1}, \cdots, f_{n-1}, p\right\}$ is a regular system of parameters for $(W \llbracket X \rrbracket)_{Q}$ and the ideal $N_{i}^{\prime}=\left(f_{1}, \cdots, f_{i}\right)(W \llbracket X \rrbracket)_{Q}$ is a prime ideal of $(W \llbracket X \rrbracket)_{Q}$ for $1 \leqq i \leqq n-1$ (cf. Corollary 1, p. 302 and Theorem 26, p. 303 of [6]). In particular, $N_{n-1}=N_{n-1}^{\prime} \cap W \llbracket X \rrbracket$ is a prime ideal of $W \llbracket X \rrbracket$ such that rank $N_{n-1}=n-1, N_{n-1} \subset Q$, and $N_{n-1} \cap W=(0)$. We now have $P_{1} \llbracket X \rrbracket=Q \cap V \llbracket X \rrbracket \supset N_{n-1} \cap V \llbracket X \rrbracket$ and $\operatorname{rank}\left(N_{n-1} \cap V \llbracket X \rrbracket\right)=$ rank $N_{n-1}=n-1$-that is, rank $P_{1} \llbracket X \rrbracket \geqq n$. Therefore, $k+1=$ $\left.\operatorname{rank} Q_{k+1} \geqq 1+\operatorname{rank} P_{1} \llbracket X \rrbracket\right) \geqq n+1$. We have already seen that $k \leqq n$, so $k=n$ and $\operatorname{rank}\left(Q_{k+1} / P_{1} \llbracket X \rrbracket=1\right.$. Thus, $P_{1} \llbracket X \rrbracket / P_{1} \llbracket X \rrbracket \subset$ $Q_{k+1} / P_{1} \llbracket X \rrbracket \subset \cdots \subset Q_{t} / P_{1} \llbracket X \rrbracket$ is a maximal chain of prime ideals in $V \llbracket X \rrbracket / P_{1} \llbracket X \rrbracket \cong U \llbracket X \rrbracket$ of length $t-k$. By assumption $t-k=(m-1) n+1$ and since $k=n$ this implies that $t=m n+1$.

We now consider the case in which $Q_{k+1}=P_{1} \llbracket X \rrbracket$. It follows from the previous argument that $n \leqq \operatorname{rank} P_{1} \llbracket X \rrbracket=\operatorname{rank} Q_{k+1}=k+1$. We will show that equality holds. Let $\mathscr{C}$ be a valuation overring of $V \llbracket X \rrbracket$ with prime ideals $(0) \subset Q_{1}^{\prime} \subset \cdots \subset Q_{k+1}^{\prime}$ such that $Q_{i}^{\prime} \cap V \llbracket X \rrbracket=Q_{i}$ 
for each $i$. Since $Q_{k} \cap V=(0)$ we may assume that $Q_{k+1}^{\prime}=\operatorname{rad}(p \mathscr{Y})$ and, by localizing if necessary, we assume that $Q_{k+1}^{\prime}$ is the maximal ideal of $\mathscr{T}$. We wish to show that $\mathscr{V} \supseteqq W \llbracket X \rrbracket$. If this is not the case then there exists $h \in W \llbracket X \rrbracket$ such that $h^{-1} \in Q_{k+1}^{\prime}$. If $f=p h$ then $f \in P_{1} \llbracket X \rrbracket, h^{-1}=p / f$, and there exists an integer $s$ such that $h^{-s}=$ $p^{s} / f^{s}=p \zeta$ for some $\zeta \in \mathscr{Y}$. But $f^{s} \in\left(P_{1} \llbracket X \rrbracket\right)^{s} \subseteq p^{s-1} P_{1} \llbracket X \rrbracket$ so we have $p \zeta=p^{s} / p^{s-1} f_{1}$ for some $f_{1} \in P_{1} \llbracket X \rrbracket$. Therefore, $1 / f_{1}=\zeta \in \mathscr{V}$ contrary to the assumption that $P_{1} \llbracket X \rrbracket \subseteq Q_{k+1}^{\prime}$. It follows that $W \llbracket X \rrbracket \subseteq \mathscr{V}$ and if $Q_{i}^{\prime \prime}=Q_{i}^{\prime} \cap W \llbracket X \rrbracket$ for $1 \leqq i \leqq k+1$ then $(0) \subset Q_{1}^{\prime \prime} \subset \cdots \subset Q_{k+1}^{\prime \prime}$ is a chain of prime ideals of $W \llbracket X \rrbracket$ such that $Q_{2}^{\prime \prime} \cap V \llbracket X \rrbracket=Q_{i}$. In particular, $Q_{k+1}^{\prime \prime} \cap V \llbracket X \rrbracket=P_{1} \llbracket X \rrbracket$. Since $[\mathscr{P}+(X)] \cap V \llbracket X \rrbracket=P_{1}+(X)$ it follows that $Q_{k+1}^{\prime \prime}$ is not maximal in $W \llbracket X \rrbracket$. Thus, $n+1>\operatorname{rank} Q_{k+1}^{\prime \prime} \geqq$ $k+1$ - that is, $k<n$. It follows that $k=n-1$ and this together with the previous argument shows that, in either case, rank $P_{1} \llbracket X \rrbracket=$ $n$. We now have that $P_{1} \llbracket X \rrbracket / P_{1} \llbracket X \rrbracket \subset Q_{k+2} / P_{1} \llbracket X \rrbracket \subset \cdots \subset Q_{t} / P_{1} \llbracket X \rrbracket$ is a maximal chain of prime ideals in $V \llbracket X \rrbracket / P_{1} \llbracket X \rrbracket \cong U \llbracket X \rrbracket$ of length $t-(k+1)=t-n$. By assumption, $t-n=(m-1) n+1$, so $t=$ $m n+1$.

REMARK. The proof of the theorem shows that if $(0)=P_{0} \subset$ $P_{1} \subset \cdots \subset P_{m}$ is the set of prime ideals of a discrete valuation $\operatorname{ring} V$ then each of the prime ideals $P_{i} \llbracket x_{1}, \cdots, x_{n} \rrbracket$ can be included in a maximal chain of prime ideals of $V \llbracket x_{1}, \cdots, x_{n} \rrbracket$ and for $0<i<m$ we have $\operatorname{rank}\left(P_{i} \llbracket x_{1}, \cdots, x_{n} \rrbracket / P_{i-1} \llbracket x_{1}, \cdots, x_{n} \rrbracket\right)=n$.

\section{REFERENCES}

1. J. T. Arnold, Krull dimension in power series rings, Trans. Amer. Math. Soc., 177 (1973), 299-304.

2. —_, Power series rings over Prüfer domains, Pacific J. Math., 44 (1973), 1-11.

3. — Algebraic extensions of power series rings, Trans. Amer. Math. Soc., to appear.

4. D. E. Fields, Dimension theory in power series rings, Pacific J. Math., 35 (1970), 601-611.

5. R. Gilmer, Multiplicative Ideal Theory, Marcel Dekker, New York, 1972.

6. O. Zariski and P. Samuel, Commutative Algebra, Vol. II, D. Van Nostrand Company, Princeton, 1960.

Received March 19, 1980 and in revised form August 25, 1980.

Verginia Polytechnic Institute and State University

Blacksburg, VA 24061 



\section{PACIFIC JOURNAL OF MATHEMATICS}

\section{EDITORS}

DONALD BABBITT (Managing Editor)

University of Galifornia

Los Angeles, California 90024

HUgo RossI

University of Utah

Salt Lake City, UT 84112

C. C. MOORE AND ANDREW OGG

University of California

Berkeley, CA 94720

\section{J. DUGUNDJI}

Department of Mathematics University of Southern California Los Angeles, California 90007

R. Finn and J. Milgram

Stanford University

Stanford, California 94305

\section{ASSOCIATE EDITORS}
R. ARENS
E. F. BECKENBACH
B. H. NeUManN
F. WOLF
K. YosHIDA

\section{SUPPORTING INSTITUTIONS}

UNIVERSITY OF ARIZONA

UNIVERSITY OF BRITISH COLUMBIA

CALIFORNIA INSTITUTE OF TECHNOLOGY

UNIVERSITY OF CALIFORNIA

MONTANA STATE UNIVERSITY

UNIVERSITY OF NEVADA, RENO

NEW MEXICO STATE UNIVERSITY

OREGON STATE UNIVERSITY
UNIVERSITY OF OREGON UNIVERSITY OF SOUTHERN CALIFONIA STANFORD UNIVERSITY UNIVERSITY OF HAWAII UNIVERSITY OF TOKYO UNIVERSITY OF UTAH WASHINGTON STATE UNIVERSITY UNIVERSITY OF WASHINGTON 


\section{Pacific Journal of Mathematics}

\section{Vol. 93, No. $1 \quad$ March, 1981}

Richard Arens, Reducing the order of a Lagrangian $\ldots \ldots \ldots \ldots \ldots \ldots \ldots$

Richard Arens, Manifestly dynamic forms in the Cartan-Hamilton treatment

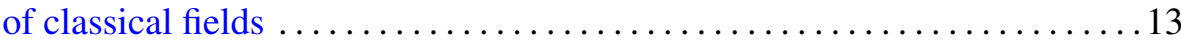

Jimmy T. Arnold, Power series rings over discrete valuation rings $\ldots \ldots \ldots 31$

Charles A. Asmuth and Joe Repka, Supercuspidal components of the

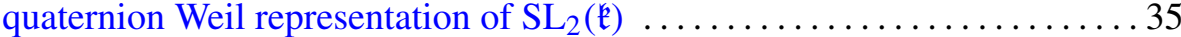

Luis A. Caffarelli and Avner Friedman, Sequential testing of several simple hypotheses for a diffusion process and the corresponding free boundary problem ................................. 49

William B. Jacob, Fans, real valuations, and hereditarily-Pythagorean fields .............................................. 95

W. J. Kim, Asymptotic properties of nonoscillatory solutions of higher order

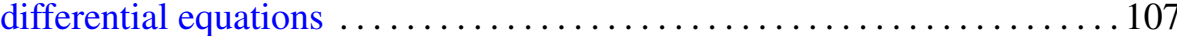

Wayne Steven Lewis, Embeddings of the pseudo-arc in $E^{2} \ldots \ldots \ldots \ldots \ldots 115$

Daniel Alan Marcus, Closed factors of normal Z-semimodules . ......... 121

Mitsuru Nakai and Leo Sario, Harmonic functionals on open Riemann surfaces ............................................. 147

John Currie Quigg, Jr., On the irreducibility of an induced

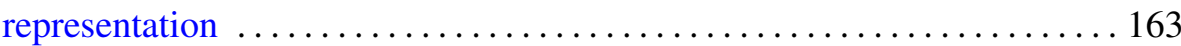

John Henry Reinoehl, Lie algebras and Hopf algebras 181

Joe Repka, Base change for tempered irreducible representations of $\mathrm{GL}(n, \mathbf{R})$

Peter John Rowley, Solubility of finite groups admitting a fixed-point-free automorphism of order $r s t$. I . . . . . . . . . . . . . . . . . . 201

Alan C. Woods, The asymmetric product of three homogeneous linear forms 